\title{
REDEFINICIÓN DE AUTORÍA Y LICENCIAS CREATIVE COMMONS: ANÁLISIS DE CONTENIDO EN BLOGS LITERARIOS
}

Ana Carolina Sampaio Coelho/Universidade de Salamanca

\begin{abstract}
RESUMEN: El presente estudio investiga los blogs literarios como una práctica cultural contemporánea. A partir de un análisis de contenido de 230 blogs elegidos de forma aleatoria, analizamos aspectos relacionados a la redefinición de autoría en Internet y la utilización de licencias Creative Commons. Por fin, investigamos si la Web 2.0 puede servir de espacio para una reconfiguración profunda en la industria de los bienes culturales.
\end{abstract}

PALABRAS CLAVE: Análisis de contenido. Autoría. Blog. Industria cultural. Creative Commons.

RESUMO: O presente estudo investiga os blogs literários como uma prática cultural contemporânea. A partir de uma análise de conteúdo de 230 blogs eleitos de forma aleatória, analisamos aspectos relacionados a redefinição da autoria na Internet e a utilização de licenças Creative Commons. Por fim, investigamos se a Web 2.0 pode ser compreendida como um espaço para uma reconfiguração profunda na indústria dos bens culturais.

PALAVRAS-CHAVE: Análise de conteúdo. Autoria. Blog. Indústria cultural. Creative Commons.

\section{INTRODUCCIÓN}

Los blogs, así como otras prácticas culturales contemporáneas mediadas por las nuevas tecnologías de la comunicación, participan de una red abierta y planetaria, la Web, y enseñan una nueva configuración social y política de lo que pasa en el mundo. Estas nuevas prácticas, propias de comunicación de la Web 2.0, al contrario de lo que sucedía con los medios de comunicación de masas, no pasan por un filtro de control sino que, los autores de blogs son creadores de múltiples contenidos con total libertad de actuación, tal como afirma Pierre Levy (2004, p. 10):

\footnotetext{
No se reinventarán los instrumentos de comunicación y del pensamiento colectivo sin reinventar la democracia, una democracia compartida en todos los lugares, activa y molecular. En ese punto de inversión total o de conclusión aventurada, la humanidad podría reestructurar su futuro. No poniendo su destino entre las manos de algún mecanismo pretendidamente inteligente, sino produciendo sistemáticamente las herramientas que le permitan constituirse en colectivos inteligentes, capaces de orientarse dentro los mares tormentosos de los cambios.
}

Surge pues una participación más activa y libre en este nuevo escenario cultural que ya no está sometido al estricto control de la industria. Para analizar las formas y los medios que la 
industria cultural puede encontrar espacio para su reconfiguración en Internet, hemos elegido analizar cómo los autores de blogs literarios enseñan en sus blogs las formas como se distribuyen sus textos literarios. Tenemos como objetivo investigar ciertos aspectos relacionados con la autoría. El carácter funcional de un blog es definido por autores como Marlow (2004), que considera el blog como un medio que destaca en el escenario por su atractivo social.

\begin{abstract}
Weblogs are a massively decentralized conversation where millions of authors write for their own audience; the conversation arises as webloggers read each other and are influenced by each others' thoughts. It is through the constant process of reading, writing and referencing that authors come to know each other at an informal level. Links are the social currency of this interaction, allowing webloggers to be aware of who is reading and commenting on their writings ${ }^{\mathrm{i}}$ (MARLOW, 2004, p. 3).
\end{abstract}

Analizaremos el fenómeno de la redefinición de la autoría y de la utilización de licencias Creative Commons (que a partir de ahora abreviaremos con las siglas CC). En blogs literarios estudiando los modos en que estos autores firman sus propios textos (ya sea a través de su nombre real, de pseudónimos o incluso mediante la ausencia de firma).

Inspirada en la filosofía del software libre, Creative Commons es el grupo de licencias que deja elegir al autor la forma en que quiere proteger su obra. El nombre de dicha licencia, que en castellano podría traducirse como "Creativos Comunes", sirve de soporte a los creadores que hasta ahora no tenían como protegerse del copyright, es decir, de los tradicionales derechos de autor.

La lógica que sostiene los principios y derechos de la propiedad intelectual debe ser urgentemente reformada para adaptarse a las circunstancias digitales actuales: se requiere una nueva protección para estos nuevos tiempos de digitalización de la cultura. Pero, para que estos cambios sean posibles, y que la cuestión del acceso al conocimiento pueda ser tratada de manera justa y democrática, las problemáticas surgidas en torno a la democratización de la economía y de la cultura deben ser tratadas de forma distinta por parte de las empresas intermediarias que permiten el acceso a los bienes culturales. Dichas empresas plantean dos posibilidades de futuro: o reinventar su negocio, creando nuevas posibilidades dentro de ese nuevo universo digital, o seguir con el discurso caduco de argumentar que quien hace uso de las nuevas tecnologías para acceder a contenidos culturales está cometiendo un crimen y no tiene ninguna ética empresarial.

Las industrias culturales deben dejar de concebir las tecnologías de comunicación como amenazas y la legislación actual tiene que empezar a garantizar el progreso en la recepción y distribución de contenidos y dejar de imponer obstáculos al libre acceso a la cultura. Pero, sobre todo, los cambios en la legislación vigente deben preservar las oportunidades para la innovación creativa y para sus nuevos ámbitos de difusión. Hoy día, existe una grieta conceptual creciente entre las leyes que regulan los derechos de autor y los usuarios de las tecnologías que permiten la libre circulación de las obras. Mientras que estas tecnologías permitan el acceso imparable a un universo cultural sin límites, las leyes lo prohíben con igual intensidad. ¿Comenzaremos a vivir bajo esta lógica de la protección exagerada y la limitación del acceso cultural? En nuestra sociedad muchas de las acciones que las nuevas generaciones realizan comúnmente, como el hecho de grabar música en su lector de mp3, serán entendidas como algo ilegal?

Así, la distribución equilibrada de bienes culturales, a caballo entre la protección de los derechos de autor y la gestión del conocimiento, está siendo uno de los caballos de batalla que precisa soluciones urgentes en este comienzo del siglo XXI. Una cosa es cierta: la lógica del conocimiento es muy distinta de la lógica que regula los bienes materiales según las leyes del 
mercado y, por lo tanto, estos dos ámbitos deben ser pensados y reformulados desde ópticas distintas.

Hasta agosto de 2010, cuando realizamos esta investigación, eran escasa las investigaciones en lo referente al uso de licencias copyleft. Por otra parte, no existía investigación alguna en lo referente a la utilización de licencias Creative Commons en el ámbito de la literatura, así como tampoco existían investigaciones respecto a la utilización de los blogs como soportes de producción y distribución de narrativas protagonizados por autores literarios. En consecuencia, consideramos que la presente investigación presenta un estudio inédito y que los datos reunidos en la misma arrojan informaciones significativas sobre la literatura digital producida actualmente en Brasil. De este modo, y para llevar a cabo el objetivo antes mencionado, hemos planteado la siguiente hipótesis:

H1: Suponemos que los autores literarios que mantienen blogs, en general, están a favor de la cultura copyleft y que poseen generalmente una licencia Creative Commons para la distribución de sus contenidos a través del blog.

\section{Metodología y especificaciones del análisis}

Para trazar el observar de las cuestiones relativas al tratamiento de la autoría, se eligió la metodología del análisis de contenido. La elección de esa metodología tiene como base la aplicación directa, estadística y cuantitativa a determinadas variables relativas al objeto de estudio de nuestra investigación: el blog literario. Hemos optado, en primer lugar, por hacer un análisis descriptivo de los componentes de los blogs literarios relativos a autoría y las licencias CC.

Una vez elegida la metodología de investigación (IGUARTUA, 2006), hemos determinado, para acotar nuestro universo de estudio, que los blogs seleccionados para la investigación deberían ser blogs brasileños colgados en los dos dominios más populares que existían en el país hasta la fecha: Blogspot y Wordpress. El éxito de su popularidad se debe a que ambos dominios poseen herramientas sencillas de edición y de publicación de contenidos. Una de las dificultades encontradas a la hora de aplicar la metodología estuvo relacionada con el tamaño de la muestra elegida, puesto que el tamaño del ciberespacio y de la blogosfera es muy volátil y no podemos contar con datos precisos ni fijos. Hemos elegido una muestra de conveniencia de 230 blogs. Además, todos ellos deben cumplir el requisito de ser blogs brasileños, colgados en los dos dominios ya mencionados. Otra condición indispensable, es que estos blogs que forman nuestra muestra deben presentar también materiales y contenidos escritos inéditos (ya se trate de poesías, cuentos o micro narrativas) para su publicación en red.

Las direcciones de los blogs que forman la muestra fueron seleccionadas de forma aleatoria. En definitiva, buscábamos blogs donde su autor colgara sus propios contenidos literarios originales e inéditos. La compilación de las direcciones de los blogs se realizó en el periodo comprendido entre mayo y junio de 2010, y los autores seleccionados debían tener sus blogs actualizados por lo menos hasta el mes de abril de 2010. 


\section{RESULTADOS}

\subsection{Datos relacionados con la identificación del autor literario}

Antes de que empecemos a detallar los resultados de esa investigación, debemos referirnos al hecho de la extrema volatilidad, modificación y el carácter efímero de los contenidos que se difunden a través de estas nuevas tecnologías digitales. La periodicidad con que los autores escriben en sus blogs no es rígida y los elementos que los componen tampoco. Más bien al contrario, la lógica del blog está ubicada en lo que llamamos la "cultura de lo efímero". Las informaciones compartidas en la red son volátiles y hay una gran dificultad en su preservación.

En general, las personas entran y salen de las redes para atender a sus expectativas y, como muchas de ellas no resultan satisfechas, tratan de cambiarlas por algo que les parezca más ventajoso. Eso se refiere a todas las variables que hemos analizado en ese estudio de análisis de contenido, con la muestra $(\mathrm{n}=230)$ de los blogs literarios brasileños colgados en los dominios de Blogspot y Wordpress. Una vez hecha esta pequeña introducción, debemos hacer constar que dichos blogs literarios fueron analizados en el período comprendido entre mayo y junio de 2010, y todas las modificaciones que se puedan observar en la versión actual de dichos blogs son fruto de procesos que ocurrieron después del periodo en que las informaciones fueron recogidas y analizadas.

Observamos que en nuestra muestra la representatividad de hombres y mujeres es muy equilibrada, con un $46,5 \%(n=107)$ de bloggers mujeres y un $43 \%(n=99)$ de bloggers hombres, mientras que el $10 \%(n=24)$ no se haya identificado por género y no hemos podido averiguar su sexo. De la muestra relativa a los 24 autores literarios no identificados por género, 5 de ellos no firman sus textos ni se identifican en ningún espacio, otros 18 forman parte de blogs literarios colectivos y 1 se identifica y firma con pseudónimo.

A partir de los datos relativos a Identificación y Firma de los bloggers, observamos que hay una tendencia mayoritaria de los bloggers que se identifican y firman con nombre propio, cerca de $61,7 \%(\mathrm{n}=142)$, seguidos por aquellos que se identifican y firman con pseudónimo, 22,2\% ( $\mathrm{n}=$ 51). En seguida observamos la presencia de bloggers que optaron por páginas colectivas, o sea, blogs colectivos, donde la identificación que prevalece es una, el nombre del colectivo, pero donde todos los autores literarios firman individualmente, un total de 9,6\% $(n=22)$ de la muestra. Hemos detectado pocos blogs literarios donde los autores no se identifican ni firman en ningún espacio, cerca de 2,2\% $(\mathrm{n}=5)$ de la muestra.

Al igual que la distribución de la frecuencia de blogs literarios en cuanto al sexo de los bloggers era muy equilibrada, también hemos observado que la forma en que los autores literarios optan por firmar e identificarse en sus textos también obedece a un equilibrio similar.

Del total de los 99 autores literarios identificados como hombres en la muestra, el $69,7 \%(n=69)$ eligen identificarse y firmar con nombre propio. Un porcentaje muy cercano a éste lo encontramos también entre las mujeres. Del total de mujeres bloggers que han participado en la investigación, el 68,2\% ( $\mathrm{n}=73$ ) también se identifican y firman con nombre propio. Entre los autores literarios hombres, $(n=23)$ el $23,2 \%$ se identifican y firman con pseudónimo. Nuevamente un número muy cercano utiliza pseudónimo en el caso de los bloggers mujeres, $(\mathrm{n}=27)$ el 25,2\%. Podemos afirmar, por lo tanto, que se trata de una tendencia y que, en este caso, el sexo no determina ningún comportamiento específico a la hora de firmar. 


\subsection{Datos relativos a las licencias Creative Commons}

Para observar cómo los autores de los blogs literarios analizados se posicionan en lo referente a los derechos de autor en la red, la primera variable que elegimos fue la de la presencia o ausencia de una licencia del Creative Commons en sus blogs. Esas licencias copyleft tratan de crear alternativas para regular las restricciones impuestas por el tradicional sistema de derechos de autor. Entre las licencias copyleft que existen, las licencias Creative Commons son las iniciativas más populares cuando se trata de trabajos creativos.

De esa manera, hemos optado por observar la proporción de blogs en la muestra elegida que poseía una de las licencias $C C$ colgadas en sus páginas. Los resultados enseñan que las licencias copyleft tienen poca representación visual entre los autores de blogs literarios. Solamente el $27,80 \%(n=64)$ de la muestra posee una licencias $C C$ colgada en el blog. De esa manera, el $72,20 \%(n=166)$ restante de la muestra no posee una licencia $C C$. La tendencia que observamos en los resultados anteriores que enseñaban un claro equilibrio en las respuestas de hombres y mujeres se mantiene también aquí.

De los 99 autores literarios hombres que componen la muestra, 26 han colgado una licencia $C C$ en su blog, o sea, cerca del 26,26\% del total de autores literarios que poseen licencia $C C$ en el blog. Del total de las 107 autoras literarias mujeres que figuran en la muestra, 33 autoras, o sea, el 30,84\% del total de autoras eligen utilizar una licencia CC. A pesar de que la presencia de esta licencia es de mayor porcentaje, el valor del Chi-cuadrado revela que la asociación entre las variables "sexo" y "presencia” no es significativa. De los autores literarios que no se identifican -y entre ellos también nos referimos a los blogs colectivos- solamente 5 bloggers utilizan licencia Creative Commons, o sea, 20,83\% del total de la muestra que posee licencias CC colgada en el blog.

De los 64 autores que utilizan licencia $C C$ en sus blogs, 41 casos se identifican y firman con nombre propio, lo que representa la elección del 64,1\% de los que poseen licencia CC. Son 16 los autores que poseen la licencia, se identifican y firman con pseudónimo, lo que representa cerca del 25\% de los autores que poseen licencia CC. Esos datos no arrojan ninguna conclusión significativa. Únicamente podemos anotar que apenas sobresale sobre el resto el número de autores que firman con nombre propio respecto a los que firman con pseudónimo y la opción elegida para utilizar las licencias obedece a la misma tendencia.

Al decidir utilizar una licencia $C C$ en su blog, el autor del trabajo sometido a licencia debe también elegir la tipología de la misma. Como ya exponemos, actualmente existen 6 tipos de licencias $C C$ y cada una posee una finalidad y una utilización específicas. Los resultados de esa investigación apuntan a que hay una concentración de trabajos licenciados en dos licencias específicas. De los 64 blogs que poseen licencias CC, 41 autores han elegido la licencia "Reconocimiento - No comercial - Sin Obra Derivada (by - nc - nd )" lo que representa un 64,1\% del total de autores que poseen licencia $C C$.

La mayoría de los autores han elegido la licencia que permite la distribución, la copia y la exhibición por parte de terceros siempre que se muestren los créditos y no se obtenga ningún beneficio comercial y tampoco se realicen obras derivadas. Se trata de una elección cautelosa que permite la licencia con ciertas reservas, toda vez que asegura un uso amplio de la obra y también permite que el autor obtenga rendimientos financieros en caso de que la obra tenga cierto éxito y reconocimiento. Otros 17 autores han elegido la licencia "Reconocimiento (by)". Este grupo corresponde al 26,56\% de autores que optaron por utilizar licencia. Al contrario de la licencia (by- 
$n c-n d$ ) que demuestra una cierta prudencia, como hemos dicho, la licencia (by) es la más permisiva de todas. A través de esa licencia, las personas pueden hacer uso comercial e igualmente pueden crear obras derivadas a partir de la obra licenciada. Lo esencial es que se haga referencia a los créditos del autor.

Vemos que, de los 22 blogs de autoría colectiva (y con firma individual) existentes en la muestra, len su gran mayoría $(n=17)$ no posee licencia $C C$, lo que representa cerca de $77,27 \%$ de los autores colectivos. Aunque la gran mayoría (el 71,13\%) de los que firman con su propio nombre tampoco registren en su blog ningún tipo de licencia Creative Commons, cerca de un 29\% (es decir, más de la cuarta parte) sí que ha utilizado alguna de estas modalidades de licencia.

Entre la muestra de estos autores que sí se identifican y firman con nombre propio, vemos que hay una predominancia por la elección de la licencia (by-nc-nd) sumando un total de 25 autores, cerca de 17,61\% del total de los firmantes, mientras que 13 autores (que representa 9,15\% de los que firman) eligen la licencia (by). Al decidir compartir sus creaciones en la Red, los autores muestran un comportamiento todavía muy prudente al permitir que la obra sea compartida: porque no permiten obras derivadas de la suya y, por lo tanto, estas obras deben ser pasadas adelante sin modificaciones.

Un caso interesante se produce entre los autores que se identifican, pero que firman con pseudónimo. De los 16 autores que eligen licencia $C C$ y firman con pseudónimo, su inmensa mayoria, 12 de los 16 (el 23,53\% del total de pseudónimos) eligen la licencia (by-nc-nd). Aunque hayan firmado con un nombre distinto al suyo, y eso pueda parecer en principio un acto de libertad en relación con la autoría del texto, percibimos que esos autores optan por proteger sus producciones con ciertas garantías para que no se haga un uso comercial con sus obras y que no estén sujetas a modificación alguna.

El número de autores literarios que han escogido otras formas de proteger sus obras, además de las licencias $C C$, es mínimo. Además de los 64 autores literarios que utilizan licencias $C C$, son apenas otros 10 autores los que eligen otras formas de protección de sus textos, o sea, que el resto de los 156 autores que componen la muestra (95,65\%) no enseñan en su blog ninguna información respecto a los derechos de autor.

Los 5 autores (2,17\%) que afirman que el texto "está sujeto expresamente a otras leyes que protegen parcial o totalmente los derechos de autor" hacen referencia a la ley de número 9.610/98 de la Constitución Brasileña relativa a los derechos de autor. Dicha ley data del 19 de febrero de 1998. Por lo tanto, esta ley es anterior al período de desarrollo y expansión de Internet y de las nuevas tecnologías de la comunicación. En el texto donde los autores se refieren a la ley, dicen que "la reproducción total o parcial, no expresamente autorizada por el autor, de cualquier texto publicado, está sujeta a las penalidades del artículo 102 de la ley”. Ese artículo dice que el titular de la obra reproducida sin permiso puede requerir la aprehensión de los ejemplares reproducidos o la suspensión de la divulgación. La ley del derecho de autor en Brasil es una de las más rigurosas en todo el mundo, donde se tipifica como crimen incluso el hecho de utilizar películas para fines educativos o la simple transferencia de la música de un CD comprado en una tienda de forma legal a un dispositivo de mp3. Actualmente, el proyecto de revisión de esa ley está siendo discutido junto al Ministerio de Cultura en Brasil, después de una serie de consultas públicas para la modernización de la ley de derechos de autor. 


\section{DISCUSIÓN}

Los resultados que hemos encontrado en el análisis de contenido de esos 230 blogs de autores literarios brasileños puede que no suponga una muestra representativa de la realidad de lo que ocurre verdaderamente en Internet respecto al tema de la investigación, pero sí que dice mucho sobre las actitudes tendenciales de los autores en la Red. Una de las grandes dificultades que hemos encontrado, como ya hemos señalado anteriormente, fue precisamente conseguir acotar el universo total de blogs específicamente literarios que existen en la blogosfera brasileña. Es imposible saber la totalidad de blogs que son utilizados con fines literarios en Brasil (y en el mundo). Buena parte de esta dificultad se debe al constante flujo de abrir y cerrar cuentas de blogs, y también debido a la ausencia de un instrumento propio y eficaz para medir la blogosfera literaria.

Cuando empezamos esta investigación, optamos por analizar el blog como práctica cultural, así que debemos acordar que muchos autores ya consagrados a través del sistema editorial tradicional han empezado a publicar sus obras utilizando la novedosa herramienta del blog, pero después han migrado hacia una página Web con dominio propio. Es por ese motivo por que esas páginas web fueron excluidas directamente de la muestra que formaba el universo del análisis, puesto que no respondían al espíritu de la herramienta del blog, en su más amplio sentido hipertextual. Es importante hacer mención a estos casos porque, en realidad, los autores consagrados que actualmente mantienen blogs o páginas Web sólo para hacer promoción de sus trabajos en un determinado momento utilizaron también el blog para publicar su obra en formato digital. Una vez esclarecidas estas cuestiones iniciales, empezaremos a analizar los resultados relativos a la redefinición del concepto de autoría, los derechos de autor en la red y el grado de aceptación de la cultura copyleft entre los blogs analizados.

Hemos visto que la cuestión de la autoría está todavía aún muy relacionada con el concepto, creado y difundido en la modernidad, de un único autor que es quien da su nombre a la obra o a los textos que firma. Del mismo modo, los conceptos de derechos de autor y propiedad intelectual, también están estrechamente ligados a la idea del autor único. La Industria Cultural actualmente refuerza aún más estos conceptos clásicos, junto con la idea de que el autor es un instrumento de valoración y clasificación de los textos. Los productos de la Industria Cultural están asociados al autor y son respaldados por su firma. Vimos que esos conceptos fueron tan férreamente establecidos y consolidados históricamente en nuestra cultura que, durante muchos años, ni siquiera fueron mínimamente cuestionados. Con la revolución de los medios y la llegada de la cultura digital, la nueva realidad nos obliga a reflexionar respecto a estos viejos conceptos. Pero observamos que, en general, estos cuestionamientos no han cambiado sustancialmente la actitud de los autores literarios con respecto a la forma escrita de producción.

El 61,7\% de los autores literarios de la muestra analizada que firman con su nombre son ejemplos que refuerzan la idea de que ese patrón de comportamiento va a seguir existiendo durante mucho tiempo. Aunque el número no sea grande ni suficientemente representativo, empezamos a ver otro tipos de trabajos literarios colaborativos, en los que sí se valen de una de las grandes fuerzas productivas de la nueva cibercultura: el hecho de compartir los contenidos y las formas de autoría. Cerca del 9,6\% $(\mathrm{n}=22)$ del conjunto de la muestra corresponde a blogs literarios colectivos, donde se reúnen algunos autores que publican sus textos. En los 22 blogs colectivos, los autores firman con sus propios nombres, pero están todos sometidos al mismo dominio del blog. Esos blogs son ejemplos de lo que Pierre Levy (2004) denomina "inteligencia colectiva”, expresión utilizada para designar una "inteligencia distribuida por todas partes”, que resulta de la unión de las competencias entre los individuos participantes. 
La idea de base de ese concepto consiste en que nadie sabe de todo, pero que toda la gente sabe de alguna cosa. A través de Internet, las personas se reúnen para compartir sus conocimientos y crear algo en común. Es importante resaltar que no hemos encontrado ningún blog colectivo con firma colectiva, es decir, que entre los blogs colaborativos no hay ningún ejemplo en el que los autores desistan de su autoría individual. La idea de la obra de autoría múltiple, abierta, fragmentada, tiene aún poca representación y presencia (por no decir nula) en la muestra analizada.

Creemos que una de las causas de la baja incidencia de proyectos colaborativos está fundamentada en la lógica de la propiedad intelectual y en los derechos de autor que dificultan procesos donde muchas fuerzas se reúnen para contribuir con algo en pro del beneficio común. Los cerca de 200 años de historia que arrastramos, correspondientes al periodo que comprende la modernidad en el que se propagó la idea de identificación del sujeto con su obra, están tan enraizados en el imaginario popular, que el proceso de adaptación a esa nueva realidad digital plural seguramente tardará un tiempo en volverse popular y socialmente aceptado.

A partir de la observación de cómo los autores se identifican y firman sus textos, podemos afirmar que la tendencia apuntada a que en la cibercultura los productos culturales creados y editados a partir de las tecnologías digitales tendrían su autoría diluida y los procesos creativos serían más abiertos, colectivos y libres, no se aplica de hecho a la realidad de los blogs literarios brasileños en el momento actual. Lo que observamos es que la autoría individual es todavía muy fuerte, así como la tendencia a crear obras cerradas y fijas, es decir, sin la colaboración de otros autores.

De esa manera, podemos afirmar que la revolución tecnológica desarrollada en los últimos años ha ofrecido a millones de autores literarios, que han abierto cuentas de blogs, un soporte de utilización sencilla que les ha ayudado a distribuir sus mensajes a todo el mundo en tiempo real.

La forma de Internet corresponde a una red llena de nudos y conexiones, de manera que nos parece que su propio lenguaje no es favorable para que la noción de la autoría permanezca igual de inamovible que en los últimos dos siglos, donde el lenguaje del soporte en papel era más propicio para que se desarrollaran trabajos lineales y de autoría única. Al alcanzar este grado de autonomía en la producción de productos culturales independientes, los autores literarios crean una actitud de confrontación con respecto a la industria cultural precedente. Por lo tanto, podríamos decir que también cuestionan el propio sistema capitalista, puesto que la mercantilización de los bienes culturales constituye una herramienta en la promoción, desarrollo y perpetuación de ese sistema económico.

Sabemos que uno de los ejes que sostiene esa misma industria es la noción de autoría. La industria cultural, personificada aquí en el papel de las editoriales, distribuidoras de cine y grabadoras, aparece ante el gran público asumiendo el papel de prescriptor, es decir, de "quien entiende del tema” $\mathrm{y}$, por lo tanto, se vende la idea de que dichas empresas son verdaderas autoridades en los asuntos culturales con los que trabajan.

Solamente el $27,8 \%(n=64)$ del total de la muestra posee una licencia CC colgada en su blog y el número de autores literarios que ha escogido otras formas de proteger sus obras, además de licencias CC, es mínimo: apenas otros 10 autores. Así, 156 autores (cerca de 67,82\%) de la muestra no muestran ningún tipo de información en sus blogs respecto a los derechos de autor. Es importante subrayar que, aunque los autores no hagan ninguna referencia expresa en sus blogs a la manera con que desean que su trabajo sea distribuido, eso no tiene que significar necesariamente que prohíban de forma expresa la copia del texto, siempre que se haga referencia al autor. Muchos 
pueden considerar que el hecho de publicar en Internet es ya per se una manera de compartir con toda la gente sus obras y esperan que se respete de forma genuina la autoría de los textos que presentan. Pero, en estos casos, no hay forma de medir esa voluntad de distribución libre a partir del análisis de contenido, puesto que la mayoría de los autores no se pronuncian expresamente en sus blogs sobre cómo quieren que sean tratados sus contenidos y cómo quieren que sean protegidos por los actuales derechos de autor en Internet.

Es interesante observar algunos puntos respecto a la utilización de las licencias CC: la licencia más utilizada es la del tipo (by-nc-nd), la que cerca el texto del autor con el mayor número de garantías posible. Los autores demuestran un comportamiento todavía muy prudente y, por regla general, no permiten que personas ajenas contribuyan en la creación de su obra de forma colaborativa. De este modo, ponemos aquí nuevamente en tela de juicio la cuestión de la existencia de trabajo colaborativo entre los autores literarios: además de que en el análisis existen pocos blogs colectivos, los autores en su mayoría tampoco permiten que la obra sea modificada. Esta característica difiere de la filosofía del software libre, por ejemplo, que tiene como principal premisa el código abierto, que permite que todos los profesionales que trabajan en la creación del software consigan contribuir y mejorar el producto informático. Del mismo modo, los blogs de autoría colectiva demuestran su desconocimiento o directamente no están de acuerdo de forma mayoritaria con la filosofía copyleft, puesto que la mayoría de ellos, el 77,2\% ( $n=17)$ de la muestra, no posee licencia $C C$.

Así que, a partir de estos resultados, vemos que, al contrario de lo que planteábamos, los autores no se muestran favorables a la cultura copyleft y a las licencias CC. La cuestión que queda aún por contestar, pero que no podemos corroborar sólo con los datos obtenidos en este análisis de contenido, es la siguiente: ¿el motivo del bajo índice de utilización de las licencias está relacionado con el desconocimiento sobre el tema o estos datos, en realidad, nos muestran que la mayoría de los autores no están de acuerdo con el sistema de licencias CC? Las grandes corporaciones y las industrias del entretenimiento se esfuerzan para trabajar en contra la diseminación que propugna la cultura libre y en cada país los representantes de esas industrias, muchos mediante acuerdos mutuos con el gobierno de turno, vienen tomando medidas distintas en ese sentido, pero siempre con el mismo objetivo: criminalizar el acto de compartir los contenidos culturales sirviéndose de las nuevas tecnologías de la comunicación. El miedo que dichas industrias tienen a perder el dominio y el control mayoritario de la distribución de los contenidos culturales las está dejando ciegas ante los cambios tan profundos que se han venido desarrollando en la sociedad desde la implementación de la cultura digital.

El discurso de las entidades que defienden a esas industrias del entretenimiento debe encontrar urgentemente una forma de negociación con la ciudadanía para proponer que las prácticas comunes de Internet (el remix, la reproducción y la creación colaborativa, por citar sólo algunas de sus posibilidades) entren en sintonía con los derechos de autor y los intereses de esas industrias. Vemos en las nuevas tecnologías de la comunicación ese potencial democrático emergente, puesto que garantizan procesos más transparentes y la participación de todos los que se conectan a la Red.

Ahora sólo falta que los autores de blogs literarios brasileños reflexionen sobre cómo este nuevo espacio, el ciberespacio donde están disponibles los bienes culturales comunes, puede llegar a cambiar lo que se entiende por economía de mercado, y cómo puede ser capaz de propugnar una nueva manera de hacer negocios y un nuevo código ético para el ejercicio de estas nuevas prácticas. Las licencias copyleft son herramientas que pueden ser utilizadas para ampliar el alcance de las creaciones de los autores. Hemos visto ejemplos de como dichas licencias han incrementado la divulgación de muchos trabajos, incluso en otros ámbitos, además del específicamente literario. 
La realidad que hemos observado a partir de este análisis de contenido nos presenta un escenario todavía poco abierto y aún no demasiado receptivo para la cultura del copyleft. En general, los resultados de esta investigación demuestran que el peso histórico de la determinación de la figura del autor y de los derechos de autor convencionales son aún más fuertes que la promesa de ausencia de mediación entre autor y lector, ostentada aún por los monopolios de las industrias de la cultura.

Queda aún lejos la promesa, al menos en literatura, de que el autor pueda convertirse también en su propio productor y beneficiarse de todos los aspectos positivos que pueden estar relacionados con eso. En la actualidad, los autores de blogs tienen en sus manos el aparato tecnológico que les permite ser revolucionarios a la hora de editar, producir y distribuir sus creaciones. Escribir otra historia distinta de la que ha sido contada recientemente por la industria de los bienes culturales, que se encontraba en manos de unas pocas y grandes industrias del entretenimiento, es la labor que los nuevos autores comprometidos deben acometer.

\section{REFERENCIAS}

IGUARTUA, Juan José. Métodos cuantitativos de investigación en comunicación. Barcelona: Bosch, 2006.

LEVY, Pierre. Inteligencia Coletiva. Para uma antropologia do espaço. São Paulo: Loyola, 2004.

MARLOW, Cameron. Audience, structure and authority in the weblog community. 54th Annual Conference of the International Communications Association. May. New Orleans, LA, 2004. 
i Los weblogs son una conversación de forma masiva descentralizada donde millones de autores escriben para su propio público; la conversación surge al tiempo que los autores de blogs se leen los unos a los otros y son influidos por los pensamientos de los demás. Es a través del proceso constante de lectura, escritura y referencia como los autores vienen a conocerse unos a otros de manera informal. Los enlaces constituyen la moneda social de esta interacción, permitiendo que los webloggers sepan quién lee y comenta sus escritos. 